\title{
Microdrill, Diode Laser, and Manual Microsurgical Stapedoplasty: A Comparative Study
}

\author{
Priit Kasenõmm, Maris Suurna \\ Department of Otorhinolaryngology, Tartu University Hospital, Tartu, Estonia \\ Email: priit.kasenomm@kliinikum.ee
}

Received November 3, 2013; revised December 1, 2013; accepted December 30, 2013

Copyright (C) 2014 Priit Kasenõmm, Maris Suurna. This is an open access article distributed under the Creative Commons Attribution License, which permits unrestricted use, distribution, and reproduction in any medium, provided the original work is properly cited. In accordance of the Creative Commons Attribution License all Copyrights (C) 2014 are reserved for SCIRP and the owner of the intellectual property Priit Kasenõmm, Maris Suurna. All Copyright @ 2014 are guarded by law and by SCIRP as a guardian.

\begin{abstract}
Background: To evaluate hearing outcome, operation time and post-operative hospital stay after primary stapedoplasty with three different techniques: manual microsurgical, microdrill-assisted, and microdrill- and laser-assisted technique. Methods: A retrospective analysis of 150 consecutive cases of primary otosclerosis was operated by one surgeon. The patients were divided into three groups depending on the utilized surgical techniques: a fully manual microsurgical stapedoplasty $(n=56)$, microdrill-assisted stapedoplasty $(n=32)$, and microdrill- and laser-assisted stapedoplasty $(n=62)$. The mean pre- and post-operative air-bone gap was calculated by using pre- and post-operative mean pure tone air- and bone-conduction thresholds at 500, 1000, 2000 and $4000 \mathrm{~Hz}$. Results: There were no statistically significant differences in the hearing outcome between the groups. The closure of air-bone gap and the improvement of the hearing were demonstrated in all study groups. Introduction of diode laser for stapes surgery resulted in significantly reduced operation time (about one-third) and the increase in the completion of surgery from $81.5 \%$ to $96.7 \%$. At the same time, the rate of complications stayed low and hospital stay dropped from three days to one day. There were no major post-operative complications in any study groups. Conclusions: Application of diode laser in stapes surgery significantly reduced operation time and increased completion rate of surgery. No statistically significant difference was found between three surgical techniques regarding hearing outcome.
\end{abstract}

\section{KEYWORDS}

Otosclerosis; Stapes Surgery; Diode Laser; Operation Time

\section{Introduction}

Stapedoplasty is one of the standard treatments advocated for the conductive hearing loss of otosclerosis. The technique originally described by Shea [1] consisted of removal of the stapes, including all or part of the footplate, and insertion of a prosthesis to reconstitute the ossicular chain. Over the years, technical innovations, primarily introduction of the small fenestra technique, and the development of modern surgical devices have contributed towards both improved outcome and safety of stapes surgery. One of the most remarkable technical innovations was the introduction of lasers in stapes surgery already three decades ago [2,3]. However, several earlier and latter studies have shown no significant differences between laser-assisted, micro-drill and manual microsurgical stapedoplasty with regard to hearing outcome [4-10]. Instead of better hearing outcome, the micro-drill and/or laser appears to be very helpful to provide greater accuracy during precise manipulations and thereby reduce surgical trauma, which is important advantages regarding patients' safety compared to handheld instruments [11-13]. More precise and less traumatic surgical techniques have great potential to reduce operation time and diminish complications. Both advantages help to save valuable medical resources.

The aim of the present study was to evaluate hearing outcome after primary stapedoplasty, operation time and the completion rate of surgery using three different techniques in our department. The occurrence of postoperative complications was also analysed. 


\section{Patients and Methods}

\subsection{Patients}

A retrospective analysis of 150 consecutive cases of primary otosclerosis treated surgically from 2000 to 2012 by a single surgeon was performed. Patients with obliterative otosclerosis, malleus and/or incus ankylosis and revision procedures were excluded from the study. Only those patients with at least a 3-month follow-up audiogram were included for analysis. The diagnosis of otosclerosis was based on a history of progressive hearing loss, negative Rinne-test, conductive hearing loss in pure-tone audiometry, normal speech discrimination, and absence of acoustic reflexes. All operations were performed under general anaesthesia. The study was performed with the approval of the Ethics Committee of the University of Tartu.

\subsection{Operative Techniques and Patients' Groups}

All operations were performed by the same surgeon (M.S.). The initial steps of the operation were the same in all cases. An endaural approach was used and a standard tympanomeatal flap was elevated to expose the middle ear. After identifying the chorda tympani nerve, bone was curetted from the postero-superior margin of the bony annulus to visualize the oval window niche. The incodostapedial joint was opened by a fine $90^{\circ}$ hook.

The patients were allocated into three different study groups according to the time period and surgical technique used. In group A (56 patients, years between 2000 and 2004), the stapedial tendon was cut manually by a sickle knife, posterior stapedial crus was separated by a hook and stapedotomy was performed by a manual perforator. In group B (32 patients, 2004-2008), the stapedial tendon and the posterior crus were cut manually, but stapedotomy was performed by a microdrill (Skeeter ${ }^{\circledR}$ Otologic Drill System, Medtronic, USA). In group C (62 patients, 2008-2012), the stapedial tendon and the posterior crus were cut by $980 \mathrm{~nm}$ diode laser (Ceralas D25, Biolitec, Germany) and stapedotomy was performed by the microdrill. The stapedial tendon and posterior crus were vaporized with the laser at power setting of $4 \mathrm{~W}$ and pulse duration of 0.1 seconds. The anterior crus was usually fractured onto the promontory for removing the stapes superstructure. If accessible, anterior crus was firstly partially vaporized with the laser.

The perforation of the footplate was typically performed in the posterior third. A manual perforator or a microdrill was used to achieve a stapedotomy about 0.5 $\mathrm{mm}$ in diameter. When creating a fenestra in the stapes footplate with the microdrill, the speed of rotation was limited to speeds under $2000 \mathrm{rpm}$ to prevent inner ear trauma. For the same reason, the laser was never used for stapedotomy. A $0.4 \mathrm{~mm}$ teflon piston with platinum wire was used in all patients. In our department, $4.25 \mathrm{~mm}$ length prosthesis is used for female patients and $4.5 \mathrm{~mm}$ for male patients. The loop of the prosthesis was clamped to the long process of the incus with a McGee forceps. The space of the oval niche around the prosthesis was sealed by small pieces of connective tissue which are harvested in the region of the endaural incision or blood. Afterwards the tympanomeatal flap was replaced, silicon sheets were put onto the incisions and the auricular canal was packed by a sponge soaked with antibiotics (e.g. Merocel). The removal of the sutures and the package was performed about one week after the surgery.

In all study groups the completion rate of surgery was calculated. After opening the middle ear, the surgeon evaluated the possibility of removing the stapes suprastructure and of stapedotomy. The evaluation and decision was made based on visual estimation according to the current skills and instrumentation.

\subsection{Follow-Up}

The patients were seen in 1 and 3 months post-operatively for a repeat audiogram. The audiological examinations were carried out using a clinical audiometer calibrated according to ISO standards. Air conduction (AC) and bone conduction (BC) thresholds were determined pre- and post-operatively. Pure tone averages (PTA) were calculated based on AC and BC threshold levels at frequencies $0.5,1,2$, and $4 \mathrm{kHz}$. Pre- and post-operative PTA air-bone gap (PTA-ABG) was calculated. The 3month post-operative AC, BC and PTA-ABG outcomes were compared to the pre-operative values.

\subsection{Statistical Analysis}

The statistical analyses were performed using the SigmaStat 2.0 (Jandel Scientific, USA) software program. Paired t-test or Wilcoxon signed-rank test was used. The threshold for statistical significance was set at $\mathrm{p}<0.05$.

\section{Results}

The post-operative audiometry showed closure of the ABG and improvement of the hearing result in all study groups (Table 1). There were no statistically significant differences in the hearing outcome between the groups. Post-operative BC-PTA decrease was small and no severe sensorineural hearing loss occurred in either of the groups. The mean pre-operative ABG was higher in the later years of the study, but the mean post-operative ABG closure tends to be better at the same time. However, these differences reached no statistical significance.

Of note was the finding that introduction of diode laser for stapes surgery resulted in significantly reduced 
Table 1. Pre- and post-operative audiological data, operation time and completion rate of surgery.

\begin{tabular}{|c|c|c|c|c|}
\hline & Group A $(n=56)$ & Group B $(\mathrm{n}=32)$ & Group C ( $\mathrm{n}=62)$ & $\mathrm{p}$ value \\
\hline Mean age, yr & 44.2 & 43.7 & 46.4 & n.s. \\
\hline Gender, \% female & 75.0 & 70.4 & 65.0 & n.s. \\
\hline Mean preop. BC, dB & 22.3 & 22.6 & 17.8 & n.s. \\
\hline Mean preop. AC, dB & 57.2 & 57.9 & 59.4 & n.s. \\
\hline Mean preop. ABG, dB & 34.9 & 35.3 & 41.6 & n.s. \\
\hline Mean postop. $\mathrm{BC}, \mathrm{dB}$ & 23.1 & 22.8 & 18.4 & n.s. \\
\hline Mean postop. AC, $\mathrm{dB}$ & 33.2 & 32.6 & 27.5 & n.s. \\
\hline Mean postop. ABG, dB & 10.1 & 9.8 & 9.1 & n.s. \\
\hline PTA-ABG < $10 \mathrm{~dB}, \%$ & 86 & 84 & 88 & n.s. \\
\hline SNHL, dB & 0 & 0 & 0 & n.s. \\
\hline Operation time, minutes & 61.8 & 64.7 & 41.3 & $\mathrm{p}<0.0001^{\mathrm{a}}$ \\
\hline Completion of surgery,\% & 78.6 & 81.5 & 96.7 & $\mathrm{p}<0.004^{\mathrm{b}}$ \\
\hline
\end{tabular}

ap value between groups A and C, and between groups B and C; ${ }^{\mathrm{b}} \mathrm{p}$ value between groups A and B vs. group C.

operation time and increased completion rate of surgery. The operation time decreased about one-third and completion rate of the surgery increased from $81.5 \%$ to $96.7 \%$ after implementation of the laser (group B vs. group C). In contrary, introduction of the micro-drill resulted in a small increase in operation time and only a slight increase in the completion rate of surgery (group A vs. group B). The number of complete surgery was 44 out of 56 in group A, 27 out of 32 in group B and 60 out of 62 in group $\mathrm{C}$. The main reasons for discontinuing the surgery was a narrow oval window niche due to dehiscent or overhanging facial nerve, promontory overhang, too wide and strong posterior stapedial crus or stapes suprastructure fixation to the promontory.

Due to insufficient documentation of post-operative symptoms in earlier paper-based medical records compared to later electronic medical records, the thorough analysis of post-operative complications was considered unreliable. The post-operative labyrinthine irritation had somewhat higher rates in earlier study period and lower rates in later of the study. The average hospital stay in group $\mathrm{A}$ and $\mathrm{B}$ was 3 days and in group $\mathrm{C}$ one day.

\section{Discussion}

The present study reaffirms the clinical safety and efficacy of lasers in stapes surgery. Although no statistically significant difference was found between the three surgical techniques regarding hearing outcome, the diode laser appeared to be the most advantageous in reducing operation time and increasing the completion rate of surgery. In average, the operation time reduced about onethird and the completion rate of stapes surgery increased about one-fifth after implementing diode laser in our department. Several studies have also demonstrated that regardless of devices used to create the stapedotomy (manual perforator, micro-drill or lasers) no significant difference in either post-operative ABG closure or postoperative sensorineural hearing loss can be found [4-10]. Some studies have shown that micro-drill is superior to manual perforator [14] and CO2-laser is superior to potassium-titanyl-phosphate (KTP) laser [15], but these improvements were not statistically significant.

Although better hearing is the most important outcome of stapes surgery, avoiding complications is not less important. Therefore, the evolution of stapes surgery and the adjunctive use of modern surgical devices, including lasers, have mostly been driven by a desire to maximise technical success and minimise complications. The most serious complication of stapes surgery is partial or complete sensorineural hearing loss, most commonly due to excessive footplate manipulation [16]. The main advantage of the lasers is that they help to reduce the surgeon's reliance on handheld instruments during precise manipulations. In the present study, there were no major complications, such as death ear, permanent facial paralysis and sustained post-operative vertigo in any of the study groups. We suggest that it could be explained by a very conservative estimation of the possibility to complete the surgery using manual instruments and discontinuing surgery whenever in doubt. It resulted, in one hand, in a considerably low completion rate of stapes surgery in the earlier study period; on the other hand, it enabled to avoid all major complications. After introduction of the micro-drill and the diode laser in later years, the surgeon's increased confidence led to a simultaneous increase in the completion rate of stapes surgery, but still kept down the complications. The main limitation of the 
present study is that the result may be influenced by the learning curve effect and surgeon's increased experience over the time. However, our surgeon had been performing stapes surgery for three years before the study. We suggest that by performing about fifty stapes surgeries annually, the learning curve was already completed. Another variable, the increase in experience over the years, could not be controlled. In addition, to avoid inter-surgeon variability, only operations performed by one surgeon were analysed.

The presence of minor post-operative complications, such as transient vertigo, tinnitus and taste disturbances, had usually low rates in all study groups. The previous studies have shown that the micro-drill and pick stapedotomy produce similar complication rates [9]. Unfortunately, we were not able to provide reliable analysis of minor post-operative complications regarding the technique used. The reason was insufficient documentation of post-operative respective symptoms in earlier paperbased medical records compared to later electronic medical records. Electronic medical records were introduced in our department as late as in 2008. However, the postoperative labyrinthine irritation causing mild or moderate vertigo and nausea seemed to have been somewhat higher incidence in the earlier and lower incidence in later study period. We suggest that decreased post-operative labyrinthine irritation may be one of the main reasons why average hospital stay dropped from 3 days to just 1 day at the end of the study.

The present study demonstrated that application of diode laser in stapes surgery significantly reduced operation time and increased completion rate of surgery. At the same time, there was no statistically significant difference between manual microsurgical, micro-drill or laser-assisted stapedoplasty with regard to hearing outcome. Our results also suggest that modern medical devices, such as the micro-drill and the diode laser, increase the precision of surgical technique and thereby reduce mechanical trauma to the vestibule. Resulting decreased labyrinthine irritation increases patients' safety and enables to reduce hospital stay.

\section{Acknowledgements}

We would like to thank Epp Pool for performing the analysis of audiological data and Pille Kool for performing statistical analyses.

\section{REFERENCES}

[1] J. J. Shea, "Fenestration of the Oval Window," Annals of Otology, Rhinology, and Laryngology, Vol. 67, No. 4, 1958, pp. 932-951.

[2] R. C. Perkins, "Laser Stapedotomy for Otosclerosis," Laryngoscope, Vol. 90, No. 2, 1980, pp. 228-241. http://dx.doi.org/10.1288/00005537-198002000-00007

[3] S. G. Lesinski and A. Palmer, "Lasers for Otosclerosis: $\mathrm{CO}_{2}$ vs. Argon and KTP-532," Laryngoscope, Vol. 99, Suppl. 46, 1989, pp. 1-8.

[4] T. M. McGee, "The Argon Laser in Surgery for Chronic ear Disease and Otosclerosis,” Laryngoscope, Vol. 93, No. 9, 1983, pp. 1177-1182. http://dx.doi.org/10.1288/00005537-198309000-00014

[5] M. Gjuric, "Microdrill versus Perforator for Stapedotomy," Clinical Otolaryngology \& Allied Sciences, Vol. 15, No. 5, 1990, pp. 411-413. http://dx.doi.org/10.1111/j.1365-2273.1990.tb00492.x

[6] J. D. Sedwick, C. L. Louden and C. Shelton, "Stapedectomy vs Stapedotomy. Do You Really Need a Laser?” Archives of Otolaryngology-Head and Neck Surgery, Vol. 123, No. 2, 1997, pp. 177-180.

http://dx.doi.org/10.1001/archotol.1997.01900020059008

[7] T. Somers, J. P. Vercruysse, A. Zarowski, M. Verstreken and E. Offeciers, "Stapedotomy with Microdrill or Carbon Dioxide Laser: Influence on Inner Ear Function,” Annals of Otology, Rhinology, and Laryngology, Vol. 115, No. 12, 2006, pp. 880-885.

[8] C. Brace, I. Keil, J. Schwitulla, K. Mantsopoulos, M. Schmid, H. Iro and J. Hornung, "Bone Conduction after Stapes Surgery: Comparison of $\mathrm{CO}_{2}$ Laser and Manual Perforator,” Otology \& Neurotology, Vol. 34, No. 5, 2013, pp. 821-826.

http://dx.doi.org/10.1097/MAO.0b013e318280dc78

[9] H. Yavuz, F. Caylakli, F. Ozer and L. N. Ozluoglu, "Reliability of Microdrill Stapedotomy: Comparison with Pick Stapedotomy,” Otology \& Neurotology, Vol. 28, No. 8, 2007, pp. 998-1001.

http://dx.doi.org/10.1097/MAO.0b013e31815a3548

[10] D. Cuda, A. Murri, P. Morchi, T. Solenghi and N. Tinelli, "Microdrill, $\mathrm{CO}_{2}$-Laser, and Piezoelectric Stapedotomy: A Comparative Study,” Otology \& Neurotology, Vol. 30, No. 8, 2009, pp. 1111-1115.

http://dx.doi.org/10.1097/MAO.0b013e3181b76b08

[11] T. Palva, “Argon Laser in Otosclerosis Surgery,” Acta Oto-laryngologica, Vol. 104, No. 1-2, 1987, pp. 153-157. http://dx.doi.org/10.3109/00016488709109061

[12] D. L. Rothbaum, J. Roy, G. D. Hager, R. H. Taylor, L. L. Whitcomb, H. W. Francis and J. K. Niparko, "Task Performance in Stapedectomy: Comparison between Surgeons of Different Experience Levels," OtolaryngologyHead and Neck Surgery, Vol. 128, No. 1, 2003, pp. 71-77. http://dx.doi.org/10.1067/mhn.2003.13

[13] S. B. Mathews, B. M. Rasgon and F. M. Byl, "Stapes Surgery in a Residency Training Program," Laryngoscope, Vol. 109, No. 1, 1999, pp. 52-53.

http://dx.doi.org/10.1097/00005537-199901000-00011

[14] C. A. Mangham Jr., "Reducing Footplate Complications in Small Fenestra Microdrill Stapedotomy,” American Journal of Otolaryngology, Vol. 14, No. 2, 1993, pp. 118121.

[15] R. Vincent, A. J. Bittermann, J. Oates, N. Sperling and W. Grolman, "KTP versus $\mathrm{CO}_{2}$ Laser Fiber Stapedotomy for Primary Otosclerosis: Results of a New Comparative Se- 
ries with the Otology-Neurotology Database,” Otology \& Neurotology, Vol. 33, No. 6, 2012, pp. 928-933.

http://dx.doi.org/10.1097/MAO.0b013e31825f24ff

[16] G. D. L. Smyth, "Immediate and Delayed Alterations in
Cochlear Function Following Stapedotomy,” Otolaryngologic Clinics of North America, Vol. 11, No. 1, 1978, pp. 105-112. 